\title{
DISCOVERING HIDDEN ARCHITECTURES OF ANCIENT TIME: 3D DATA SURVEY TO REVEAL THE MYTH OF MITHRA IN SANTA MARIA CAPUA VETERE
}

\author{
M. Campi ${ }^{a}$, A. di $\operatorname{Luggo}^{a}$, R. Catuogno ${ }^{a}$, D. Iovane ${ }^{a}$, D. Palomba ${ }^{a}$ \\ ${ }^{a}$ DIARC, Department of Architecture - URBAN/ECO, Interdepartmental Research Center University of Naples FEDERICO II \\ campi@unina.it, adiluggo@unina.it,raffaele.catuogno@gmail.com,daniela_palomba@virgilio.it, domenico.iovane@ virgilio.it
}

Commission WG V/2

KEY WORDS: Databases System, Cultural Heritage, Virtual Archaeology, 3d modelling Mithraea, Laser scanner

\begin{abstract}
:
The research illustrated in this paper is part of a wider testing ground, aimed at identifying and developing processes of le arning and development taking place in archaeological contexts. These sites are investigated through the use of advanced technology, based on integrated methods of survey of new generation. The aim is to identify alternative languages of representation, able to read and to represent effectively the analyzed object and to propose alternative enjoyment systems. The case study is the Mithraeum in Santa Maria Capua Vetere, for which were carried out surveys with non-contact techniques, based on the use of instrumentations, for the acquisition of colorimetric and metric informations, that exploit a non-invasive approach. Data coming from laser scanner's sensors, combined with GPS receivers, are processed to define a three-dimensional centimetric space, in a single reference system of individual scans. Purpose of this research is to identify representation techniques able to communicate the sense of the multiple interrelations and of interferences among parties, providing the subsequent insertion of data into a wider project of cataloging, fruition and valuation of cultural heritage. The chance to diffuse Digital surveys is now a possible and implementable reality. The purpose is to spread awareness of World Heritage, in a participatory manner and based on the spirit of knowledge, conveying all the strength of cultural content that heritage has.
\end{abstract}

\section{INTRODUCTION}

Restlessness, bewilderment, curiosity and wonder are the feelings of those who investigate and work to discovery the sites whose origins refer to ancient times. They are antique areas where an unavoidable curiosity leads to research the original configuration and to the knowledge of rites and customs of distant and past realities. Archaeological sites, discoveries and findings reveal and talk about ancient cultures full of multiple suggestions that become field of research, knowledge and experimentation of new communicative and informative processes of analysis.

As part of a larger research program, aimed at defining innovative research methodologies for the knowledge, appreciation and enjoyment of cultural heritage, we can identify in archaeological heritage the privileged testing ground; the architectonic resource, subject to an elaboration process, becomes knowledge through the establishment of its representative model. The valorization is one of the fundamental aspects for the preservation of the architectonic resource, a process that moves through its understanding and that is also aimed at identifying "forgotten" rather than hidden realities: memory of ancient civilizations.

The case study illustrated in this paper, is part of a wider testing ground whose aim is the knowledge of "unique architectures": the Italian Mithraea. The particular object from the past, topic of this paper, is the Mithraeum of Santa Maria Capua Vetere, in the ancient Capua, already famous for its precious archaeological findings of huge value and quality and for this called by Cicero the "second Rome". It is a suggestive place that describe, in a dimension halfway among history, myth and legend, ancient civilizations and the mystical, secret and initiatic cult that they practiced inside the Mithraea.
These are the places consecrated to the God Mithra, godhead with oriental origin, whose cult spread in the western civilization from the 1th century b.C.. These environments date back mainly to the Roman Hellenistic period and are mostly underground sites that find their size in natural cavities, in caves carved into tuff or rock, rather than in environments realized as a reproduction of grottos, generally with a rectangular plant and covered by a barrel vault. The Spelaeum was the place reserved to the worship of Mithra, a long hall, often preceded by a vestibule, characterized by a central corridor and on the sides two praesepia, benches or podia, inclined toward the wall, designed to accommodate the agape, the ritual banquet, and where believers sat during sacred ceremonies. All the focus was on the altar, in the back of the hall, and on the painted or, more often, sculptural iconography, that reproduced the tauroctony, the killing of the sacred bull in the hands of God: a mythological scene full of symbolisms and meanings.

\section{THE CASE STUDY}

Placed at $4,70 \mathrm{~m}$ below the street level, the Mithraeum of Santa Maria Capua Vetere is reachable thanks to a stair with fornix and a flight of steps, with a slight slope, created in 1932 to allow the access to the archaeological site. It's here that you can enter inside another dimension, in a dark and mysterious environment dating back to a period between the late 2 nd and the early 3 th century a.D., which evokes ancient rites where the only connection with the outside world was represented by the three openings obtained in the side of the vault, today occluded. The underground environment, discovered in 1922, once was near the ancient Capitolium, the main forum of the 


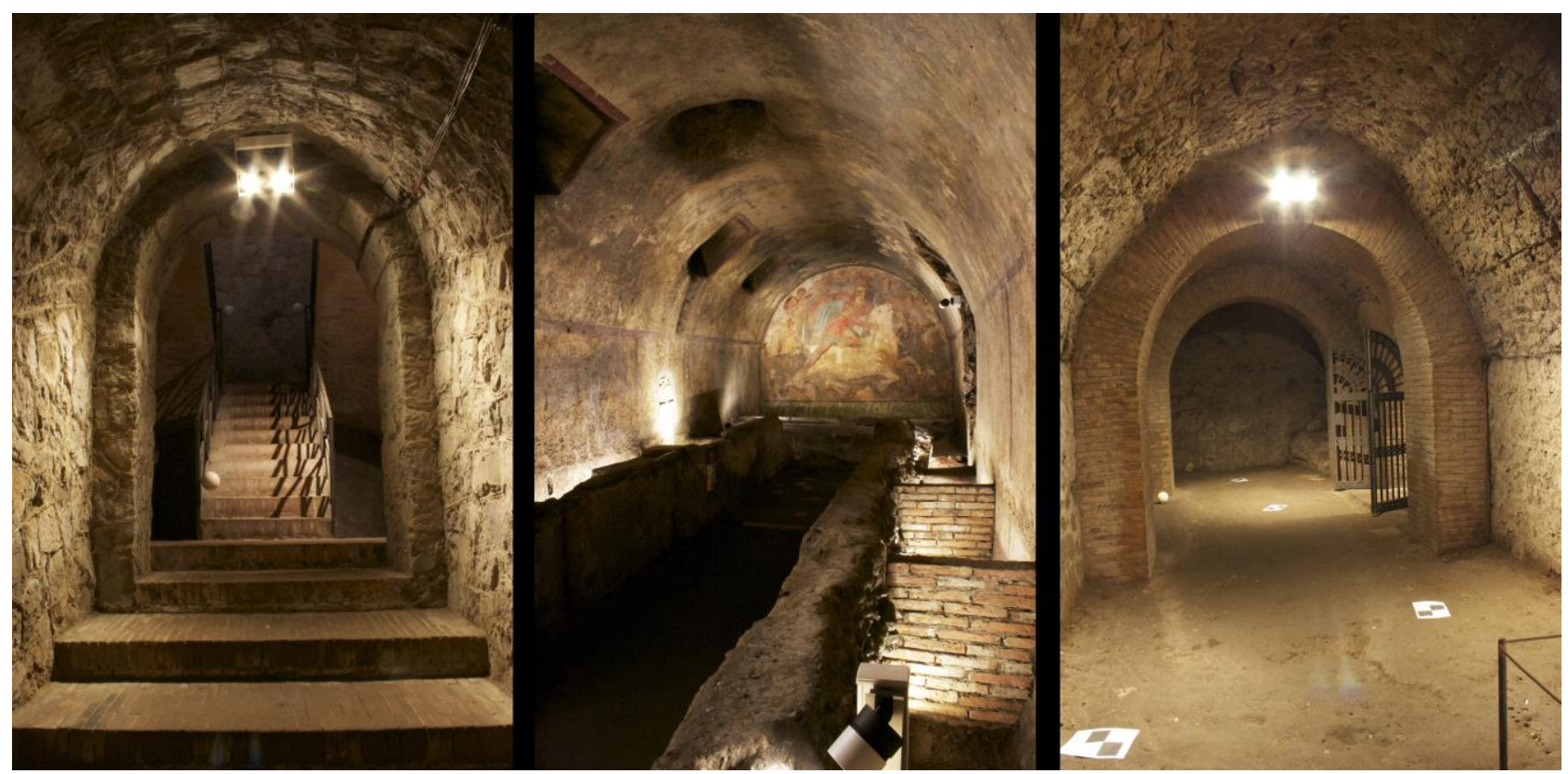

Figure 1. Mithraeum of Santa Maria Capua Vetere

Capuana metropolis, and consists of two galleries, orthogonally arranged to draw a space with an $\mathrm{L}$ shape plant. The original entrance, identified on the south side of the first tunnel that you cross also today, allowed entering into a cryptoporticus which served as a vestibulum, tripartite today after consolidation works with the realization of two large brick arches. As in the stereotyped solution, from the vestibule you can enter into the spelaeum. This is also conceived as a long rectangular and lengthened room, of about $12.20 \times 3,50$ meters, covered by a vaulted decorated ceiling with star motifs with eight tips, alternating blue and red ones, on a yellow background, symbolizing the sky under which they carry out the initiatory path. The nuggets of glass paste, included in the center of decorative elements of the vault, produced interesting light effects created by light coming from the torches. Unlike the vestibule, this environment is fully plastered and painted. Purple red bands draw, on the long walls, some quadratures. To underline the access to the sacred environment we can find two frescoes, opposite each other: two figures representing the dadophoroi in oriental clothes placed in front of an altar on which burns a bid, interpreted as a personification of Sol Oriens, the one with the torch raised, and the Sol Occidens, with the torch down. The oldest praesepia, still visible, developed only for a length of 1.25 meters and they were less high than those of the later period were. The latters, realized almost on the total length of the hall, were found lacking of the horizontal plane and they draw the central corridor characterized, next to the altar, by a flooring in opus signinum with fragments of marble slabs. Some quadratures in red bands also mark the front of the low walls of podia, where we can find scenes from the Mithraic initiation, divided into fourteen scenes equally arranged between the two podia, facing each other. Among the scenes still readable, we can identify the initiated man, depicted naked and kneeling and flanked by mystagogus and teletarcha, respectively the help of the officiant and the implementer of the rite. However, what makes this site a place of singular beauty and uniqueness, as well as his conservation status over the centuries, is the Taurocedio's fresco, made for the altar of spelaeum. To her is dedicated the entire back wall, which insists on the brick altar characterized by a red stucco coating and a channel that runs on the entire front side. The iconography is faithful to the traditional one, where the young Mithra realizes the sacrifice of the bull. The scene takes place in front of a cave and there are several characters with significantly symbolic and allegorical value. The representation is characterized by brilliant and varied colors, contrasting with the image of the white bull, around which are arranged the protagonists of the story. The dog and the snake, but also the scorpion, depicted while stings the bull, the two dadophoroi, the luminaries, the Sun and the Moon, are in contrast with the godheads Oceanus and Tellus Mater. The Mithraeum's iconographic cycle includes a final testimony represented on the wall opposite to the tauroctona representation, dedicated to the image of the goddess Moon, portrayed on a chariot pulled by two horses.

\section{METHODOLOGY}

Survey operations carried out in the framework of these studies have confirmed and validated the interpretation of historians, referring to the precise orientation of Mithraeum's halls in Santa Maria Capua Vetere. A regular geometry characterizes the arrangement of the two environments that follow the orientation of the cardinal points: the west-east direction is chosen for the spelaeum, and the north-south for the vestibulum.

As part of this research, it was developed a survey protocol for the knowledge of the archaeological heritage, using advanced technologies of the integrated survey of new generation and new techniques of representation, able to allow reading and representing effectively particular archaeological sites. The measurements were carried out using non-contact techniques, based on the use of instrumentations with laser sensors for the acquisition of colorimetric and metric informations. In order to obtain a non-invasive survey, working on an archaeological heritage, was used a laser scanner in combination with GPS receivers for defining a centimetric three-dimensional system as single reference of several individual scans. The adoption of laser scanner three-dimensional techniques is able to achieve a geo-morphometric database that has shown to be particularly 

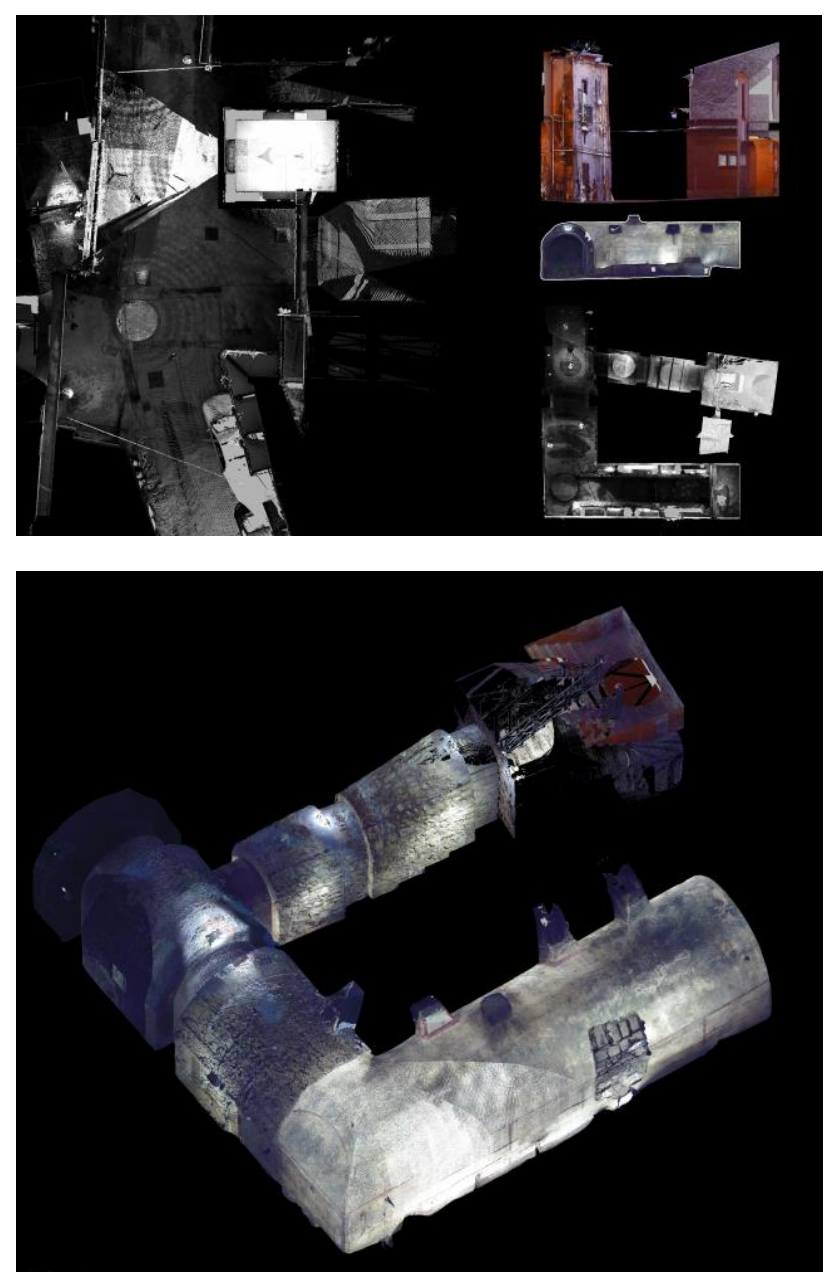

Figure $2-3$. Views of the digital model of the point cloud

effective and useful for the study and knowledge of the underground areas. Implosive dimensions that realize themselves in the substance that surrounds it. Places where the spatialities are designed as "strenght of remove" in which the empty, the cavity, the inside becomes the researched size, that one of the fulfillment of the scene. Conflicting dimensions, created with the antithesis between internal and external dimension. In fact possible it is to generate a 3D model, the point cloud, which is a faithful reproduction of it, through which investigate the morphological and metrological character of the site. A model that establishes an analogy with the real model, of which it becomes mimesis, able to represent and reproduce, in a full way, specificities and peculiarities. A mathematical model, reproducible, obtainable from the definition in a continuous sequence of sections/profiles, which allows the development of a surface, generated by interpolation of the curves obtained by the model of the point cloud. The experience is that one to represent / to process / to control / to know a vacuum, unlike the common practice that through the representation of buildings configures the "empty". The integrated use of a topographic survey, carried out with a total station and GPS, permitted the geo-referencing of the points clouds, acquired with the use of a laser scanner, allowing the control and the knowledge of the orientation as well as to define its consistency in relation to the context in which is it located. The information acquired, matched to 3D modelling functionalities, allowed to reach more complete representation methods, able to transmit the sense of multiple interactions and interference among parts and the consequent data input into a wider project of cataloging, fruition and valuation of cultural heritage that now is in progress. The chance to disseminate the Digital findings is now a reality and can be implemented, in order to diffuse the awareness of World Heritage in a participatory way and based on the spirit of knowledge of the heritage and cultural content that it conveys. Therefore the fruition of this architecture can be well supported by innovative communication technologies, accompanied by multimedia spaces which allow, in a virtual environment, the experience of the visit of Mithraeum, both in the current configuration that in an hypothetical original aspect. In the specific field of archaeology it is now possible to refer to a specific sector of Virtual Archaeology, that includes the study and the investigation of archaeological sites, virtually rebuilt and managed through augmented reality. It also realizes a new product of interaction, in which the cultural heritage, after a critical processing, turns itself into a usable and accessible knowledge. However, virtuality is also reflected in the construction of an image as a representation of the "double" of reality, a representation and a construction of a revelatory image of a different reality that is realized in the threedimensional model of the point cloud. The presentation of the site is realized through the development of a hybrid image, a mimetic, cognitive and investigative image of the studied and promoted site. At the three-dimensional model of the point cloud are flanked two-dimensional images extracted from it (orthophotos), used as a communicative and descriptive tool in the phase of preparation and realization of the drawings, with a strong evocative value particularly effective for the scope.

\subsection{The archaeological survey}

Retracing the time backwards allows us to rediscover the treasures of the past, meticulous work and scientifically completed by that discipline which is represented by "'archeology". The ability to retrace the time spent, thanks to an operation supported by a number of theories, methodologies and procedures, is achieved in the fulfillment of the relationship analysis-survey. An area in which the structures, object of the study, can not be understood and investigated in the absence of a survey. In this way it is not anymore intended to be as a simple measurement of a building or an urban context, but on the contrary, conducted rigorously and scientifically, it leads to a deep and complete understanding of

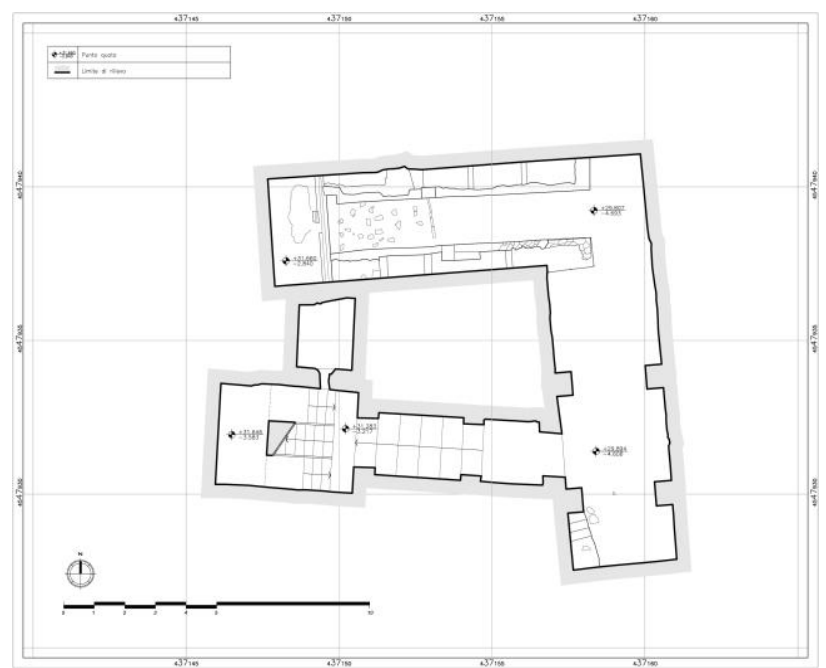

Figure 4. Graphic restitution of the plan of Mithraeum 


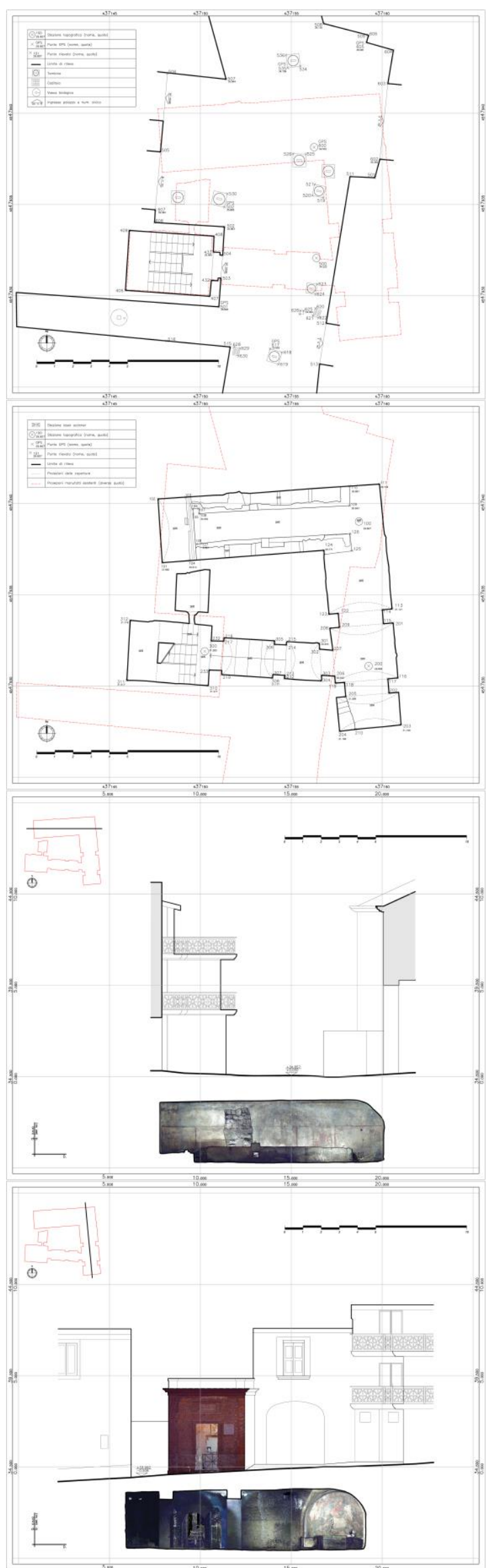

Figure 5. Graphics processing Mithraeum, plans and sections the object. In this regard, we can talk about an archeology of architecture, where is investigated the history of the buildings, reconstructed with an archaeological method, based on an analysis of the materials used and of the techniques created to build them, as well as describing the effects of time on their walls. In archeology we usually have to deal with findings in the form of artifacts, or rather man-made object or territories resulting from the union of man and nature, that become essential documents of study. The representation, through conventional signs of the survey phases, is not only a diagnostic reading, tool of analysis and control, effective nondestructive investigation, but also is a powerful tool for the historical-critical understanding. The archaeological drawing always has moved in parallel, since its inception, with topographic inquiries or better with topographic surveys, so the excavation phases are strongly related to the phases of surveying and at the same time to their graphical representation. Finally, we must not forget the contribution of digital technologies, which has allowed the progress of topographic and structural survey, not only in the field of representation through the tool of the drawing, but also in the stratigraphic reading of the analyzed objects. The drawing, that reproduces reality through the graphic paraphrase, has as its goal to make it possible to compare, triggering a process that takes place in the practice of comparison. Therefore, the surveyor is a researcher, to whom is entrusted the task of analyzing carefully the archaeological context on which he is acting, through agreed practical, in the construction of the data and in their interpretation. The phases of a survey, in their complexity, in some cases wrongly devalued, are undervalued considering their full potential, not only in research field, but also in that one of communication. The archaeological survey differs in some cases from any other type of survey for some components, due to the variety of objects that we can find on the site and the resulting graphical representation, which is why the archaeological survey has a particular methodological approach that intends to investigate something hidden. Basically, the procedures of surveying in the archaeological field, except for some special cases, do not differ from those used for recent architectural artifacts. The working methods in recent years have been deeply updated, by using new technologies and more sophisticated instruments, that enable a fast acquisition of a large number of data. This methodology has now become an indispensable requirement. The product of the archaeological survey is its graphical representation. In fact, this is not only a critical analysis of the object, but it becomes its own illustration, or rather is the philological and scientifically agreed basis of the archaeological illustration: the place where the research is intertwined with a good disclosure, where art and literature describe of men, monuments and landscapes without sacrificing the story, where you can exercise at the best the celebrative skill of archeology. The richness and complexity of archaeological sites generate the need to survey and to document as much as possible, going from the general to the particular.

\subsection{Objectives}

The use of a laser scanner gives you the chance to acquire, in a very short time, a large amount of spatial data, obtaining the survey of every object, a small building like a part of the city, with a three-dimensional grid of points from which it is possible to reconstruct the perfect geometry of the studied object, returned through the "point cloud". The sensor, in 


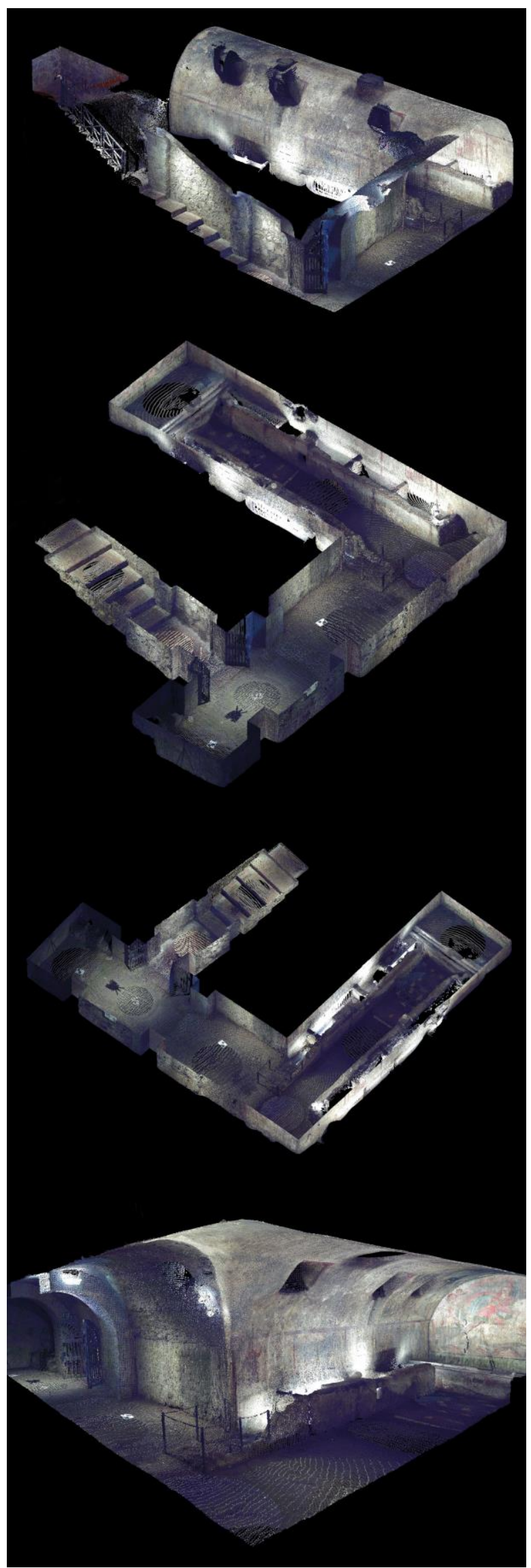

Figure 6. The cloud model has been developed to highlight different features of the site addition to the topology data, for each laser pulse emitted by the scanner, also acquires the value of reflectance. This value allows distinguishing the different materials available and it's particularly effective in the field of archeology; the combination of the sensor data and a camera, often integrated, allows then to color the points of the cloud. From the data processing we can extract:

- a "colored" point cloud with the values of reflectance or RGB values acquired by the camera;

- the textured mesh (the point cloud is triangulated by generating a three-dimensional model, on which is mapped the acquired image coming from the camera);

- sections and profiles:

- the three-dimensional model (from which it is possible to obtain surfaces, volumes, sections, elevations, etc.. documenting in this way the survey).

The acquired data and the construction of a cognitive model allow using the 3D laser scanner in several different fields of application:

- to check the progress of documentation activities during the several interventions phases carried out by the operators, verification about the condition of erosion and degradation due to weather conditions or caused by visitors;

- analysis of the yard in a non-invasive way to carry out measurements. Implementation of plans, sections and elevations 2D;

- to carry out three-dimensional models for the use of the site in remote mode;

- database for cataloging and to access to information.

\subsection{Terrestrial laser scanning}

The survey carried out using a laser scanning systems can be defined as a technique that allows obtaining qualitative and quantitative informations of an object or area, through the analysis of data acquired by a remote device, the sensor. This sensor is not in contact with the artifact and allows reorganizing the flow and the methods of data acquisition, of recording, of analysis and of data storage. The threedimensional model, obtained by laser scanning, becomes a data bank from which to extrapolate morphological and geometric informations, data on construction materials and also, with a particularly high resolution, it can be transformed into a model of analysis of degradation phenomena. From the "point cloud" is then possible to extract plans, sections and elevations of the archaeological site under consideration. The survey carried out using digital technology, Terrestrial Laser Scanning (Terrestrial Laser Scanning $=$ TLS) is a fast and precise application in several fields, like, for example, land surveying, architecture, industry, medicine and archeology. The advantages of this system are the reliability of the measurement and the speed of data acquisition, as well as the reduction of working time, and the ability to highlight some aspects, that traditional surveying techniques also permit, but with a lower accuracy or with a greater use of time. Supported by classical methods of surveying of an archaeological site, it allows to study, with reduced approximations, the site of interest and to have data extremely versatile for the preparation of a documentation of excavation:

- to build sections and plans of a portion of the archaeological site;

- to construct three-dimensional models of complex or articulated structures;

- the calculation of areas and volumes of excavation; 


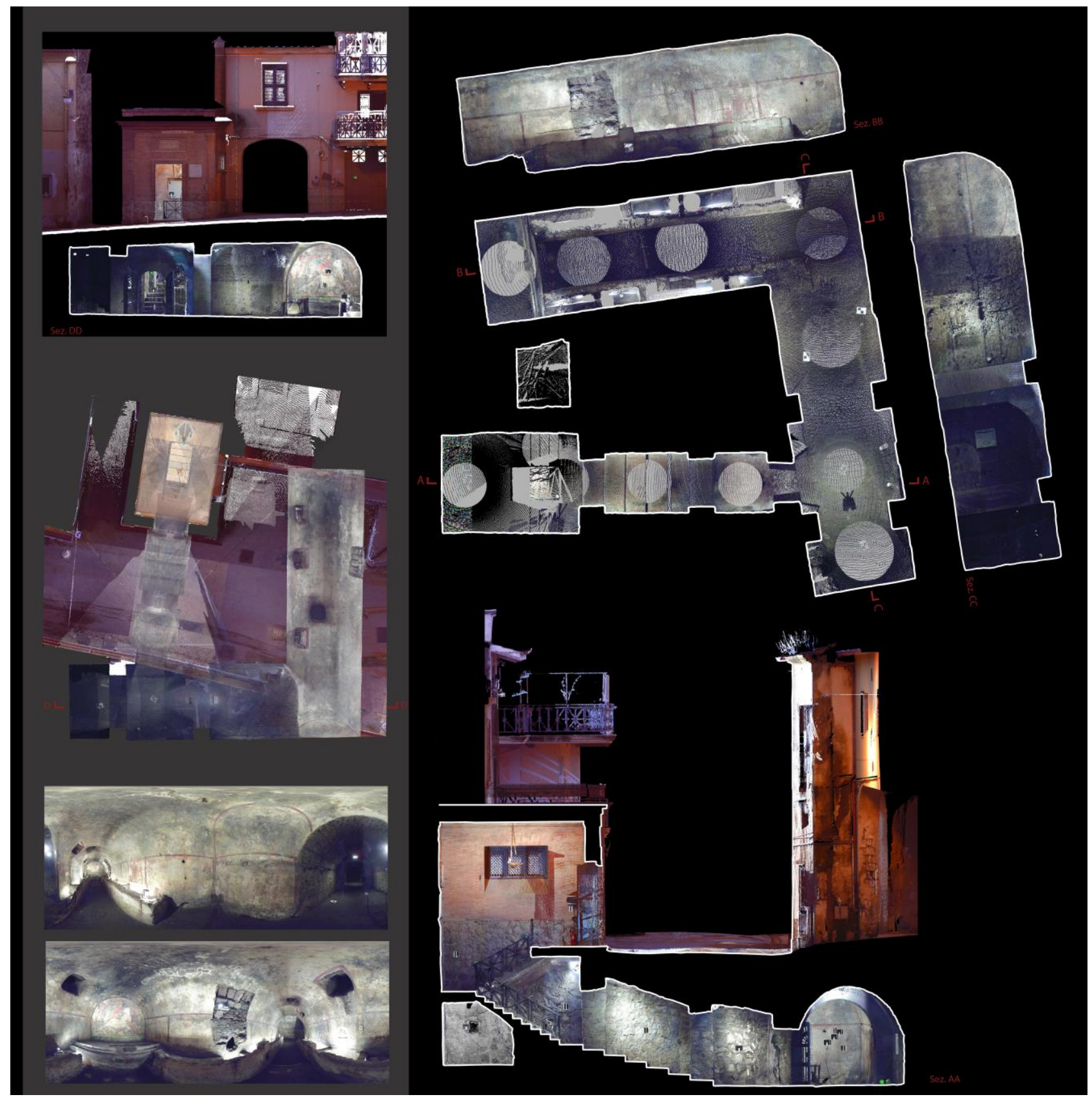

Figure 7. Orthophotos and photos spherical of the point cloud. Plans and sections

- data conversion for different software platforms to use for any type of drawing.

\subsection{Instrumentation used}

The instrumentation used for surveying is constituted by a laser scanner FARO Focus 3D. Its sensor has a measurement range of the nominal distance between $60 \mathrm{~cm}$ and 120 meters, with an accuracy in the measurement of the distance in the order of $\pm 2 \mathrm{~mm}$ to 25 meters and an angular resolution of $0.009^{\circ}$. The vertical visual field is of $305^{\circ}$, the horizontal visual field of $360^{\circ}$ and the speed of acquisition of points is amounting to 976,000 points / sec. It carries out the measurement by analyzing the phase of the signal and it stores the data on a removable memory inside the machine. The scanning parameters can be adjusted through a touch screen display integrated into the instrument. This one allows working with the tool even without the aid of a computer. Moreover it's possible, through the supplied software, Faro Scene, to create some configuration files on the PC to transfer to the SD card and then load them from the scanner. Another interesting possibility is the chance to have a wireless link with a computer, which makes the tool manageable to distance. Compared with other types of scanner, it has a contained weight ( $5 \mathrm{~kg}$ including the internal battery), which allows a fast operation in the field, requiring for commissioning station a photographic tripod instead of a topographical one, and thanks to the presence of a compensator is internal able to measure the vertical direction, facilitating the entire operation. A digital camera inside the instrument, with optical axis that is 


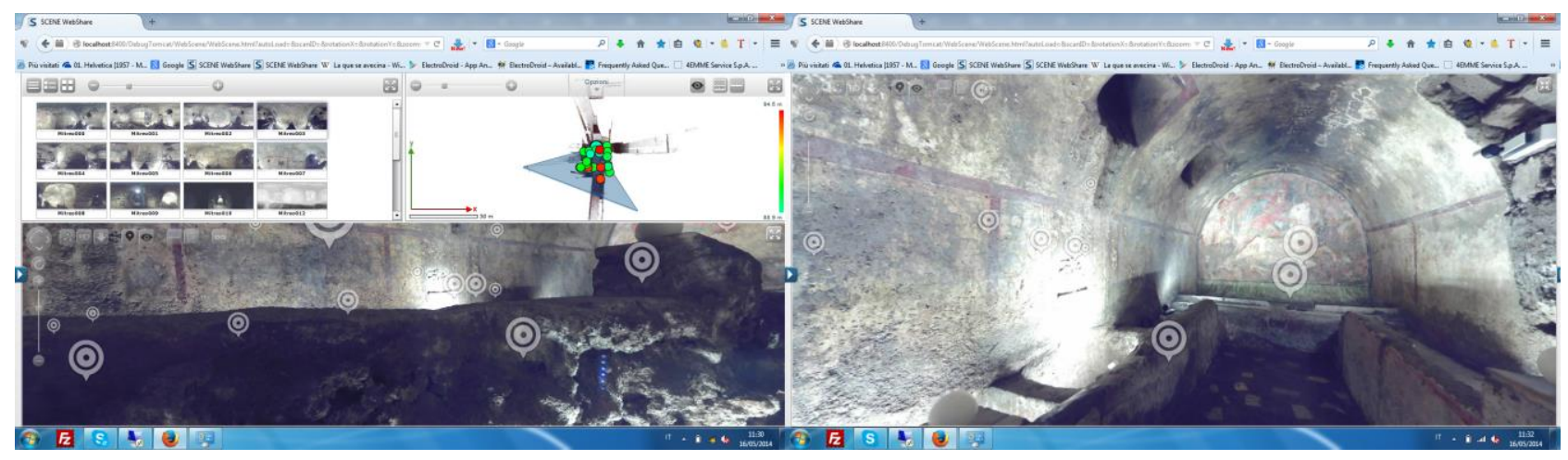

Figure 8. Images of Faro web-Share, software made available by Faro for the use of content on the network.

coaxial with the measuring beam of the laser, allows coloring each point acquired with a three-dimensional RGB color value taken from the photograph.

\subsection{Operative phases of survey and data processing}

Examined the morphology of the object of the survey, 24 scans were acquired to cover the entire site, with useful step of acquisition of $6 \mathrm{~mm}$, using 6 and 7 balls and checkerboard targets for the georeferencing of individual clouds. The result was a "cloud" containing information concerning the three coordinates $\mathrm{X}, \mathrm{Y}, \mathrm{Z}$, and the RGB color, acquired through the camera of the scanner. After this phase of acquisition on field, data were processed with the Faro Scene software, through the construction of the roto-translation matrix of individual scans, carried out identifying three common points (spheres or target) or two common points with the aid of an inclinometer between adjacent scans. The next step was to export to the united cloud in pod size, to be processed with the software Pointools Berkeley and then to proceed to the analysis of the model through dynamic sections and thematic coloration. In the same time, were realized the orthophotos (plans and sections), importing the point clouds in fls format directly in the software of the PointCab GmbH. We discovered that this last software was very versatile and interesting for the administration of the points. In fact, the construction of plans of projection is realized in the plant simply by drawing a section line, in addition to the quality of representation of the orthophotos, significantly different from other more expensive software, also in terms of computational resources.

\subsection{Networking of the model}

Once we had completed the entire union and management of the scans, we decided to put the digital model in networking, storing it on a server and using as format of data exchange E57, as protocol for querying the Tomcat database. For the management instead of both sides, server-customers, we used Faro web-Share, a software made available by Faro for the use of content on the network. The querying has been done using a plant of the studied site, automatically generated from Faro Scene, with the position of the station points of the scanner, which refer to the individual scans.

\subsection{Total station and GPS}

To provide an appropriate cognitive apparatus, were used different survey methods, in order to obtain a threedimensional numerical model. Particularly, we used a total station with subsequent implementation of satellite instruments. The surveying of Mithraeum was performed with Total Station Topcon GPT-7000, equipped with a high degree of accuracy. The use of the Station in Earth's topography is particularly useful for a precise planimetric surveying of the archaeological structures. After the acquisition of points, data were post-processed (to obtain poligonal vector layers, quoted points and lines) and geo-referenced thanks to cornerstones acquired with satellite methodology. In this case, we used 6 Stations Levelling for a total of 220 points detected. The survey with the total station allowed to determine for each point some spatial coordinates, related to a local system, and then to transform and conform them to a global reference system of satellite surveying. The satellite surveying, related to the field of archeology, has in recent years become an essential tool for the study of sites, and it aims not only to define the global reference system but also to determinate the invariant control points in different representation scales. The surveying of Mithraeum was carried out with GNSS mode (Global Navigation Satellite Systems) acronym that includes GPS satellite systems (United States), GLONASS (Russian Fed) and Galileo (the European Community, under construction), allowing you to provide positioning services with a high degree of accuracies. The spatial determination of land points $(\lambda, \varphi, H$ or $\mathrm{X}, \mathrm{Y}, \mathrm{Q})$, extended to the whole planet, is carried out through measurements of lengths and times provided by satellites into orbital navigation. The surveying system used, through the only use of the receiver, has the most advanced satellite positioning technology. The receiver used, equipped with an innovative technology, brings together all three satellite positioning systems. The signals, planned for the modernization of GPS and GLONASS satellite systems, create a system designed to capture all satellite positioning signals present and future. The determination of points coordinates are expressed, through the initial calculation or any processing procedures, in different reference systems or geodetic datum, including, that one taken as a reference: WGS84 (World Geodetic System 1984). The different satellite systems can be used simultaneously, allowing obvious advantages in areas where the visibility of the sky is limited by natural or artificial obstacles. The phases of surveying are divided into two sections: the first, with the involvement of the entire area, the second, consisting in finding points of station, subsequently used for detecting horizontal and vertical alignment by using a total station. The plano-altimetric data collection was performed using a Topcon HiPer-Pro GNSS RTK receiver, fully integrated, base and mobile, while the acquisition of the data was carried out using a handheld computer equipped with 


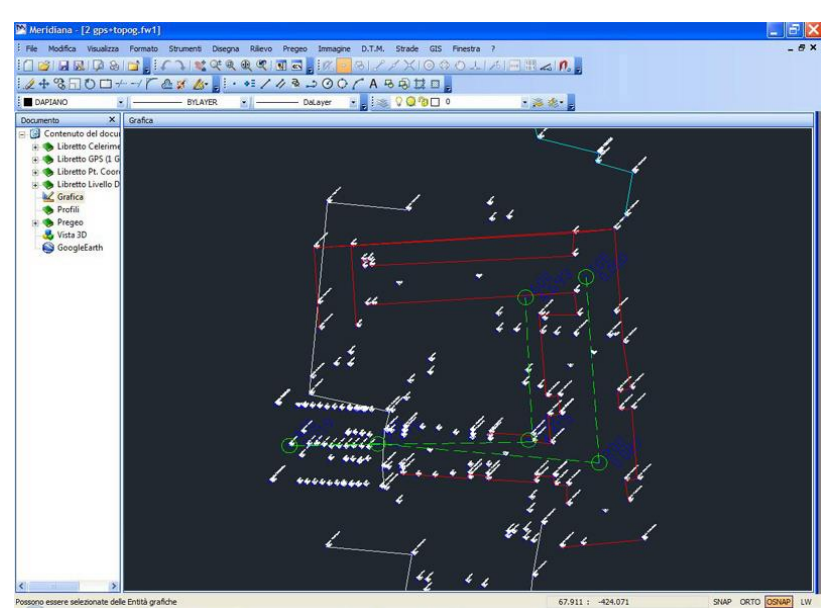

Figure 9. Orientation of the stations referred to a local system and then anchored to points of orientation.

software named Mercury. The latter, is a program for the acquisition and management of data acquired on the field. It combines ease of use, completeness in calculations, and a CAD to manage graphical data that makes easier, more intuitive and faster, surveying operations. The software enables the acquisition of data in RTK mode with the help of a modem (radio or GSM), with Network of Permanent Stations VRS and in post-processing mode. The acquisition of points is done in a simple and fast way, being always available the opportunity to check the number of satellites and their geometric arrangement on the Sky Plot, the precisions with values of PDOP, the calculation of the ambiguity, the quality of radio signal, and a series of detailed information on the individual satellites, tracked by the receiver. Mercury provides different modes of acquisition of the point. In these phases, it was possible to insert additional notes and descriptions of the points in an editable archive.

The phases of surveying have been performed by ensuring that: - the number of satellites simultaneously received by the station during a session was never lower than 6 ;

- that no observed satellite had an elevation of less than 15 degrees.

Data processing enabled then the classification in the WGS84 system, determining their position and making their readings in the international system. At this point, we carried out the calculation of coordinate transformation from the WGS84 to the Nazionale Rome 40 system; the coordinate calculation Gauss-Boaga, in automatic mode, was performed with the help of the program of coordinate transformation, VERTO IGM. Finally, the elevation framework was related to the Regional Network GNSS of Campania, to which the program Verto was reported for both leveling and as a base for the satellite survey.

\section{CONCLUSIONS}

In this context, we verified and tested the potential of modern Geomatics techniques (advantages and limitations, integrability, etc.).

Experimentation has the purpose to delineate a context in which to define methodologies and guidelines for the Cultural Heritage's surveying, necessary to systematize an activity often fragmented and not guided by scientific criteria in the choice of techniques, technologies, procedures, of the final products most appropriate in very different situations. The work described

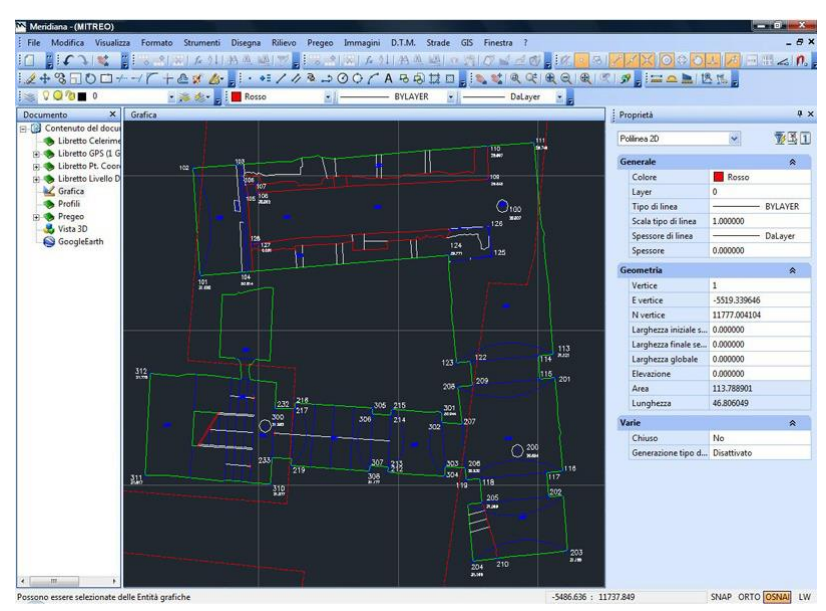

Figure 10. Graphical display within the CAD environment dedicated and enriched by specific topographic features.

above refers above all to techniques for surveying with total station, satellite surveying and laser scannering, but it is part of a larger research oriented towards the integration of multiple Geomatics techniques. Especially, about the comparison and the relationship among total station, satellite surveying and laser scannering, we want to underline the richness that an integrated approach among different methods of survey can lead, in terms of accuracy and readability of the final products. The survey with the Station and satellite surveying can help the understanding of laser data and facilitate the cleaning of noise and of those data which can not be deleted automatically by the software: the chance to texturize the model with oriented photo of the object, allow to combine metric and descriptive datum. A multi-disciplinary approach is the future for the knowledge and for the surveying of Cultural Heritage. The use of a single system of reference for data, coming from different techniques (photogrammetry, GPS, remote sensing, laser scanner) allows the reading and understanding of each object of interest, not only as a special case, but also in the context to which it belongs, increasing the possibilities for its study and its understanding. Finally, the study suggests the combined use and especially of compensation for these instruments, above all in terms of final results and useful products for the study of several problems. The use of a laser scanner technology in an archaeological survey allowed to analyze and obtain more accurate information and in a very short time, compared to those that would be obtained with a traditional survey methodology. This methodology allowed, not only a fast data acquisition, with related processing in a short time, but also the possibility to take advantage of the acquired data for different purposes, such as the realization of a virtual reconstruction.

The different laser technologies such as TOF (time-of-fly), Phase Shift and Triangulation, correctly integrated, would allow a LOD fruition (level of detail) to proceed from the general to the particular, optimizing the hardware for the management of the final product and allowing the creation of a general map of finds in an archaeological database. Knowledge and protection, enhancement and preservation of the memory, are the strong reasons that lead toward the enjoyment for the whole community of this heritage of data, full of information potentiality. The road is that one that goes toward the definition of "new images", a new iconographic heritage, and memory of significant configurations. 